\title{
LACUNARY MÜNTZ SYSTEMS
}

\section{by PETER BORWEIN and TAMÁS ERDÉLYI}

(Received 12th April 1991)

The classical theorem of Müntz and Szász says that the span of

$$
\left\{1, x^{\lambda_{1}}, x^{\lambda_{2}}, \ldots\right\}, \quad 0<\lambda_{1}<\lambda_{2}<\cdots \rightarrow \infty
$$

is dense in $C[0,1]$ in the uniform norm if and only if $\sum_{i=1}^{\infty} 1 / \lambda_{i}=\infty$. We prove that, if $\left\{\lambda_{i}\right\}$ is lacunary, we can replace the underlying interval $[0,1]$ by any set of positive measure. The key to the proof is the establishment of a bounded Remez-type inequality for lacunary Müntz systems. Namely if $A \subset[0,1]$ and its Lebesgue measure $\mu(A)$ is at least $\varepsilon>0$ then

$$
\left|a_{0}\right| \leqq c\left\|\sum_{i=0}^{n} a_{i} x^{\lambda_{i}}\right\|_{A}
$$

where $c$ depends only on $\varepsilon$ and $\Lambda$ (not on $n$ and $A$ ) and where $\Lambda:=\inf _{i} \lambda_{i+1} / \lambda_{i}>1$.

1991 Mathematics subject classification: 41A50.

\section{Introduction}

A very beautiful theorem of Müntz and Szász says that

$$
M:=\operatorname{span}\left\{1, x^{\lambda_{1}}, x^{\lambda_{2}}, \ldots\right\}, \quad 0<\lambda_{1}<\lambda_{2}<\cdots \rightarrow \infty
$$

is dense in $C[0,1]$ in the uniform norm if and only if

$$
\sum_{i=1}^{\infty} \frac{1}{\lambda_{i}}=\infty
$$

This is very much a theorem about continuous functions on intervals. So it can be proved that exactly the same theorem holds in $C[A]$ provided

$$
A \subset[0, \infty)
$$

is a closed set with non-empty interior. This result is due to Clarkson and Erdös [5]. When $\bar{A}$ has no interior it is by no means obvious what happens. Our intention is to prove the following theorem. 
Theorem 1. Suppose $\lambda_{i} \geqq \Lambda^{i}(i=1,2, \ldots)$, where $\Lambda>1$, and suppose $A \subset[0, \infty)$ is any set with positive Lebesgue measure. Then

$$
M:=\operatorname{span}\left\{1, x^{\lambda_{1}}, x^{\lambda_{2}}, \ldots\right\}
$$

fails to be dense in $C[A]$ in the uniform norm.

Indeed, under the above assumptions, if $y \in A$ is a point of Lebesgue density 1 then every function $f$ from the uniform closure of $M$ on $A$ is of the form

$$
f(x)=\sum_{i=0}^{\infty} a_{i} x^{\lambda_{i}}, \quad x \in[0, y) \cap A
$$

where $\lambda_{0}=0$. This in turn rests on the following inequality.

Inequality 1 (Remez-type inequality). Suppose $\rho>0$ and $A \subset[\rho, 1)$ is a closed set of measure $\varepsilon>0$. Suppose $\lambda_{0}=0$ and $\lambda_{i} \geqq \Lambda^{i}(i=1,2, \ldots)$, where $\Lambda>1$. Then

$$
\left\|\sum_{i=0}^{n} a_{i} x^{\lambda_{i}}\right\|_{[0, \rho]} \leqq c\left\|\sum_{i=0}^{n} a_{i} x^{\lambda_{i}}\right\|_{A}
$$

where the constant $c$ depends only on $\rho, \varepsilon$ and $\Lambda$ (and not on $n$ and $A$ ).

Here, and in what follows $\|\cdot\|_{A}$ denotes the uniform norm on $A$.

In a seminal paper [5], Clarkson and Erdös prove Theorem 1 in the case where $A:=[1-\varepsilon, 1]$. The fact that Inequality 1 holds in this interval case is critical to our argument. This follows from [5] and is proved in Section 3. (These interval results are more generally applicable to any system where $\sum_{i=1}^{\infty} 1 / \lambda_{i}<\infty$.)

Proofs of the Müntz-Szász Theorem may be found in [4], [7] and [8] with various generalizations and extensions in $[2,3,10,12,13$, and 16]. A discussion of Remez-type inequalities for polynomials is given in [6].

Our proof relies on an examination of (generalized) Chebyshev polynomials from $M$. In particular we must establish estimates for the size of their zeros. This is done in Section 2. The very close relationship between the location of zeros of the associated Chebyshev polynomials and the possibility of approximation is discussed in [1] and [2].

Section 3 contains a proof of the Remez-type inequality for $\operatorname{span}\left\{1, x^{\lambda}, x^{\lambda^{2}}, \ldots\right\}$. In the fourth section we offer a comparison theorem which allows us to extend our results to Müntz spaces

$$
M:=\operatorname{span}\left\{1, x^{\lambda_{1}}, x^{\lambda_{2}}, \ldots\right\}
$$

where for some $\Lambda>1$, 


$$
\lambda_{i} \geqq \Lambda^{i}, \quad i=1,2, \ldots
$$

Section 5 contains an example which shows that a bounded Remez-type inequality for an infinite Müntz system cannot hold on arbitrary perfect sites of measure 0 .

In the final section we characterize the Müntz systems which are dense in $C[A]$ in the uniform norm for every countable closed $A \subset[0,1] .\left(\sum_{i=1}^{\infty} 1 / \lambda_{i}=\infty\right.$ is necessary and sufficient, assuming $\lambda_{0}=0$ and $\inf _{i}\left(\lambda_{i+1}-\lambda_{i}\right)>0$.)

\section{Zeros of Chebyshev polynomials}

The generalized Chebyshev polynomial from

$$
M_{n}:=\operatorname{span}\left\{x^{\lambda_{0}}, \ldots, x^{\lambda_{n}}\right\}, \quad 0 \leqq \lambda_{0}<\lambda_{1}<\cdots
$$

with respect to a compact set $A \subset[0, \infty)$ is denoted by

$$
T_{n}(x):=T_{n}\left\{\left[\lambda_{0}, \ldots, \lambda_{n}\right]: A\right\}(x)
$$

and is defined to be

$$
T_{n}(x):=c\left(x^{\lambda_{n}}+\sum_{i=0}^{n-1} a_{i} x^{\lambda_{i}}\right)
$$

where we chose $\left\{a_{i}\right\}_{i=1}^{n}$ to minimize

$$
\left\|x^{\lambda_{n}}+\sum_{i=0}^{n-1} a_{i} x^{\lambda_{i}}\right\|_{A}
$$

and $c$ is chosen so that

$$
\left\|T_{n}\right\|_{A}=1 \text { and } \quad \lim _{x \rightarrow \infty} T_{n}(x)=+\infty
$$

Then, $T_{n}$ achieves $\pm \max _{x \in A}\left|T_{n}(x)\right| n+1$ times in $A$ with alternating sign and has exactly $n$ zeros in $(0, \infty)$. Suppose always $0 \leqq \lambda_{0}<\lambda_{1}<\cdots$.

Lemma 1. Let $0 \leqq \alpha<\beta$ and $1 \leqq m \leqq n$. Then the positive real zeros of

$$
T_{n-m}\left\{\left[0, \lambda_{m+1}, \lambda_{m+2}, \ldots, \lambda_{n}\right]:[\alpha, \beta]\right\}
$$

and

$$
T_{n-m+1}\left\{\left[0, \lambda_{m}, \lambda_{m+1}, \ldots, \lambda_{n}\right]:[\alpha, \beta]\right\}
$$


interlace. In particular, the smallest positive real zero of $T_{n-m+1}$ is smaller than the smallest positive real zero of $T_{n-m}$.

Proof. Consider $T_{n-m+1}-T_{n-m}$. The argument is a straightforward counting of zeros.

Lemma 2. Suppose $\lambda_{i} \leqq \gamma_{i}, i=1, \ldots, n$, with strict inequality at least once. Then the smallest positive real zero of $T_{n}\left\{\left[0, \lambda_{1}, \ldots, \lambda_{n}\right]:[0, \beta]\right\}$ is smaller than the smallest positive real zero of $T_{n}\left\{\left[0, \gamma_{1}, \ldots, \gamma_{n}\right]:[0, \beta]\right\}$.

Proof. See [3, Proposition 1].

Lemma 3. If $0 \leqq m \leqq n$ and

$$
T_{n-m}\left\{\left[0, \lambda_{m+1}, \lambda_{m+2}, \ldots, \lambda_{n}\right]:[0, \beta]\right\}
$$

has all its zeros in $[\alpha, \beta]$ then

$$
T_{n}\left\{\left[0, \lambda_{1}, \lambda_{2}, \ldots, \lambda_{n}\right]:[0, \beta]\right\}
$$

has at most $m$ zeros in $(0, \alpha)$.

Proof. This follows from Lemma 1.

Lemma 4. Suppose $\sum_{i=1}^{\infty} 1 / \lambda_{i}<\infty$ and $\inf _{i}\left(\lambda_{i+1}-\lambda_{i}\right)>0$. Then there exists constant $c>0$ independent of $n$ so that the smallest positive real zero of $T_{n}\left\{\left[0, \lambda_{1}, \ldots, \lambda_{n}\right]:[0,1]\right\}$ is greater than $c$.

Proof. If $\lambda_{1} \geqq 1, \sum_{i=1}^{\infty} 1 / \lambda_{i}<\infty$ and $\inf _{i}\left(\lambda_{i+1}-\lambda_{i}\right)>0$, then we have the Markov-type inequality

$$
\max _{0 \leqq x \leqq 1-\varepsilon}\left|p^{\prime}(x)\right| \leqq \eta\left(\varepsilon,\left\{\lambda_{i}\right\}\right) \max _{0 \leqq x \leqq 1}|p(x)|
$$

for every $p \in M_{n}, n \in \mathbb{N}$, and $0<\varepsilon<1$, where the constant $\eta\left(\varepsilon,\left\{\lambda_{i}\right\}\right)$ depends only on $\varepsilon$ and the sequence $\left\{\lambda_{i}\right\}$. This was obtained in [1] based on the results of Clarkson and Erdös [5]. Now the lemma follows from the equioscillation of $T_{n}$, the Mean Value Theorem, and the above Markov-type inequality. When $0<\lambda_{1}<1$ the scaling $x \rightarrow x^{1 / \lambda_{1}}$ gives the desired result from the already proved case.

Lemma 5. Suppose $\sum_{i=1}^{\infty} 1 / \lambda_{i}<\infty$ and $\lambda_{i-1} / \lambda_{i}(i=2,3, \ldots)$ is nondecreasing. Then there exists a constant $c$ depending only on the sequence $\left\{\lambda_{i}\right\}$ so that

$$
T_{n-m}\left\{\left[0, \lambda_{m+1}, \lambda_{m+2}, \ldots, \lambda_{n}\right]:[0,1]\right\}
$$


has all its positive real zeros in $\left[1-c \lambda_{n-m} / \lambda_{n}, 1\right]$.

Proof. Note that the assumptions of the lemma imply $\inf _{i}\left(\lambda_{i+1}-\lambda_{i}\right)>0$. Let $\beta_{m, n}$ be the smallest non-negative real zero of

$$
S_{m, n}:=T_{n-m}\left\{\left[0, \lambda_{m+1}, \lambda_{m+2}, \ldots, \lambda_{n}\right]:[0,1]\right\}
$$

Then consider, for $n>m \geqq 0$,

$$
U_{m, n}(x):=S_{m, n}\left(x^{\delta_{m, n}}\right)
$$

where

$$
\delta_{m, n}:=\max _{n \geqq i \geqq m+1} \frac{\lambda_{i-1}}{\lambda_{i}}=\frac{\lambda_{n-1}}{\lambda_{n}}
$$

Note that

$$
U_{m, n}=T_{n-m}\left\{\left[0,\left(\delta_{m, n} \lambda_{m+1}\right),\left(\delta_{m, n} \lambda_{m+2}\right), \ldots,\left(\delta_{m, n} \lambda_{n}\right)\right]:[0,1]\right\}
$$

and

$$
\lambda_{h-1} \leqq \lambda_{h} \delta_{m, n}<\lambda_{h} \quad \text { if } \quad m+1 \leqq h \leqq n .
$$

So by Lemma 2 the smallest positive real zero of $U_{m, n}(x)$ (which is just $\beta_{m, n}^{1 / \delta_{m, n}}$ ) is greater than the smallest positive real zero of

$$
T_{n-m}\left\{\left[0, \lambda_{m}, \lambda_{m+1}, \ldots, \lambda_{n-1}\right]:[0,1]\right\}
$$

So in particular,

$$
\begin{aligned}
\beta_{m, n} & >\beta_{m-1, n-1}^{\delta_{m, n}} \\
& \vdots \\
& >\left(\beta_{0, n-m}\right)^{\delta_{m, n} \cdot \delta_{m-1, n-1} \cdots \delta_{1, n-m+1}} .
\end{aligned}
$$

Here, by Lemma 4,

$$
1>\beta_{0, n-m}>c_{1}>0
$$

since $\beta_{0, n-m}$ is just the smallest root of

$$
T_{n-m}\left\{\left[0, \lambda_{1}, \ldots, \lambda_{n-m}\right]:[0,1]\right\}
$$

So 


$$
\begin{aligned}
\beta_{m, n} & >c_{1}^{\delta_{m, n} \cdot \delta_{m-1, n-1} \cdots \delta_{1, n-m+1}} \\
& =c_{1}^{\left(\lambda_{n-1} / \lambda_{n}\right) \cdot\left(\lambda_{n-2} / \lambda_{n-1}\right) \cdots\left(\lambda_{n-m} / \lambda_{n-m+1}\right)} \\
& =c_{1}^{\lambda_{n-m} / \lambda_{n}}
\end{aligned}
$$

Also, by the Mean Value Theorem, for every $c_{1}>0$ there exists a $c_{2}>0$ so that

$$
c_{1}^{\delta} \geqq 1-c_{2} \delta
$$

for $0<\delta<1$. Therefore

$$
\beta_{m, n} \geqq 1-\frac{c_{2} \lambda_{n-m}}{\lambda_{n}}
$$

as required.

From Lemmas 3 and 5 we deduce:

Lemma 6. Suppose $1 \leqq \lambda_{1}<\lambda_{2}, \ldots, \sum_{i=1}^{\infty} 1 / \lambda_{i}<\infty, \lambda_{i-1} / \lambda_{i}(i=2,3, \ldots)$ is nondecreasing, and $0 \leqq m \leqq n$. Then there exists a constant $c$ depending only on the sequence $\left\{\lambda_{i}\right\}$ so that

$$
T_{n}:=T_{n}\left\{\left[0, \lambda_{1}, \ldots, \lambda_{n}\right]:[0,1]\right\}
$$

has at most $m$ zeros in the interval

$$
\left(0,1-\frac{c \lambda_{n-m}}{\lambda_{n}}\right)
$$

and at least $n-m$ zeros in

$$
\left[1-\frac{c \lambda_{n-m}}{\lambda_{n}}, 1\right]
$$

\section{The Lacunary case}

In most of this section let

$$
M:=\operatorname{span}\left\{1, x^{\lambda}, x^{\lambda^{2}} \ldots\right\}, \lambda>1
$$

and

$$
M_{n}:=\operatorname{span}\left\{1, x^{\lambda}, \ldots, x^{\lambda^{n}}\right\}
$$


Lemma 6 gives the following as a special case.

Lemma 7. $T_{n}:=T_{n}\left\{\left[0, \lambda, \ldots, \lambda^{n}\right]:[0,1]\right\}$ has at least $n-m$ zeros in

$$
\left[1-\frac{c}{\lambda^{m}}, 1\right]
$$

and at most $m$ zeros in $\left(0,1-c / \lambda^{m}\right)$ where $c>0$ depends only on $\lambda$.

Let $P_{n}$ be the Chebyshev polynomial from $M_{n}$ on a fixed, compact $A \subset[0,1]$ of measure at least $\varepsilon>0$. Since $P_{n}$ is the Chebyshev polynomial from $M_{n}$ on $\widetilde{A}:=$ $\left\{x \in[0,1]:\left|P_{n}(x)\right| \leqq 1\right\}$ as well, we may, without loss, assume that $A$ is comprised of at most $n$ disjoint intervals. Choose $\delta>1$ so that

Now partition $[0,1]$ into subintervals

$$
\sum_{k=1}^{\infty} \frac{1}{\delta^{k}}=\varepsilon
$$

$$
\begin{array}{cc}
I_{1}:=\left[0, \beta_{1}\right] \quad \text { with } \quad \mu\left(I_{1} \cap A\right)=\frac{1}{\delta}, \\
I_{2}:=\left[\beta_{1}, \beta_{2}\right] \quad \text { with } \quad \mu\left(I_{2} \cap A\right)=\frac{1}{\delta^{2}}, \\
\vdots \\
I_{n-1}:=\left[\beta_{n-2}, \beta_{n-1}\right] \quad \text { with } \quad \mu\left(I_{n-1} \cap A\right)=\frac{1}{\delta^{n-1}}, \\
I_{n}:=\left[\beta_{n-1}, 1\right] \quad \text { with } \quad \mu\left(I_{n} \cap A\right)>\frac{1}{\delta^{n}},
\end{array}
$$

where $\mu$ denotes the Lebesgue measure.

Lemma 8. Suppose $j, 2 \leqq j \leqq n$, is fixed and $A \cap I_{j}$ contains an interval of length $\Delta_{j}$. Then there are positive constants $c_{\lambda}$ and $c_{\lambda}^{\prime}$ depending only on $\lambda$ so that $A \cap I_{j-1}$ contains an interval of length $\Delta_{j-1}$ where

$$
\Delta_{j-1}>\frac{c_{\lambda}}{\delta^{j}\left|\log \Delta_{j}\right|}
$$


whenever $0<\Delta_{j}<c_{\lambda}^{\prime}$.

Proof. Let $\left[a_{j}, b_{j}\right]$ be the interval of length $\Delta_{j}$ in $A \cap I_{j}$ and choose $m_{j} \in \mathbb{R}$ so that $\Delta_{j}=c / \lambda^{m_{j}}$, where $c$ is as in Lemma 7. Consider the Chebyshev polynomial $T_{n}$ from $M_{n}$ on the interval $\left[0, b_{j}\right]$. From Lemma 7 , by the scaling $x \rightarrow b_{j} x$ we can deduce that $T_{n}$ has at least $n-m$ zeros in $\left[a_{j}, b_{j}\right]$ where $m$ is the smallest nonnegative integer not less than $m_{j}$. In particular $P_{n}$ (the Chebyshev polynomial from $M_{n}$ on $A$ ) has at most $m+2$ zeros in $\left(0, a_{j}\right]$, otherwise $T_{n}-P_{n} \in M_{n}$ would have more than $n$ zeros in $\left(0, b_{j}\right]$ (counting every positive zero without sign change twice). It follows that $A \cap I_{j-1}$ is the union of at most $m+4$ intervals and hence $A \cap I_{j-1}$ contains an interval of length at least

$$
\frac{1}{\delta^{j-1}(m+4)} \geqq \frac{1}{\delta^{j-1}\left(\frac{\log c-\log \Delta_{j}}{\log \lambda}+5\right)} \geqq \frac{c_{\lambda}}{\delta^{j}\left|\log \Delta_{j}\right|}
$$

whenever $0<\Delta_{j} \leqq c_{\lambda}^{\prime}$ with some positive constants $c_{\lambda}$ and $c_{\lambda}^{\prime}$ depending only on $\lambda$.

Lemma 9. Let $\delta>1$ and $c_{\lambda}>0$ be as in Lemma 8. Consider the (backwards) iteration

$$
\Delta_{k-1}:=\frac{c_{\lambda}}{\delta^{k}\left|\log \Delta_{k}\right|} \quad \text { where } \quad \Delta_{n}=\frac{1}{n^{2} \delta^{n}}
$$

Then there is a constant $c_{\lambda, \delta}$ depending only on $\lambda$ and $\delta$ so that

$$
\frac{1}{k^{2} \delta^{k}}<\Delta_{k}<\frac{1}{2}
$$

whenever $c_{\lambda, \delta}<k \leqq n$.

Proof. Suppose

$$
\frac{1}{2}>\Delta_{k}>\frac{1}{k^{2} \delta^{k}}
$$

Then

$$
\begin{aligned}
\frac{1}{2} \Delta_{k-1} & =\frac{c_{\lambda}}{\delta^{k}\left|\log \Delta_{k}\right|}>\frac{c_{\lambda}}{\delta^{k}\left|\log \left(k^{2} \delta^{k}\right)\right|} \\
& \geqq \frac{c_{\lambda} / \delta}{k(|\log \delta|+2|\log k|) \delta^{k-1}}
\end{aligned}
$$




$$
\geqq \frac{1}{(k-1)^{2} \delta^{k-1}}
$$

provided $k>c_{\lambda, \delta}$ with a constant $c_{\lambda, \delta}$ depending only on $\lambda$ and $\delta$. The result now follows.

We will need the following inequality that may be found in $[14, \mathrm{p} .54]$. We include a distinct new proof.

Inequality 1 in the Interval Case.

Suppose $\lambda_{0}=0, \inf _{i}\left(\lambda_{i+1}-\lambda_{i}\right)>0$ and

$$
\sum_{i=1}^{\infty} \frac{1}{\lambda_{i}}<\infty
$$

Then, for every $\varepsilon \in(0,1)$,

$$
\left\|\sum_{i=0}^{n} a_{i} x^{\lambda_{i}}\right\|_{[0,1]} \leqq c_{\varepsilon}\left\|\sum_{i=0}^{n} a_{i} x^{\lambda_{i}}\right\|_{[1-\varepsilon, 1]}
$$

where $c_{\varepsilon}$ depends on the sequence $\left\{\lambda_{i}\right\}$ and $\varepsilon$ but not on $n$.

Proof. Let $\varepsilon \in(0,1)$ be fixed. First let $\lambda_{1} \geqq 1$. Assume indirectly that there are $p_{m} \in M$ so that $A_{m}:=\max _{0 \leqq x \leqq 1}\left|p_{m}(x)\right| \rightarrow \infty$, while $\left\|p_{m}\right\|_{[1-\varepsilon, 1]}=1$. Let $q_{m}:=A_{m}^{-1} p_{m}$. Then, without loss, we may assume that

$$
\max _{0 \leqq x \leqq 1}\left|q_{m}\right|=\max _{0 \leqq x \leqq 1-\varepsilon}\left|q_{m}\right|, \quad m=1,2, \ldots
$$

As in [1, Lemma 2] (see also the proof of Lemma 4), for every $0<\varepsilon^{\prime}<1$ we have

$$
\max _{0 \leqq x \leqq 1-\varepsilon^{\prime}}\left|q_{m}^{\prime}(x)\right| \leqq \eta\left(\varepsilon^{\prime}\right)
$$

where $\eta\left(\varepsilon^{\prime}\right)$ is a constant depending only on $\varepsilon^{\prime}$. Therefore $\left\{q_{m}\right\}_{m=1}^{\infty}$ is a sequence of uniformly bounded equicontinuous functions on every closed subinterval of $[0,1)$, hence by the Arzela-Ascoli Theorem there is a subsequence of $\left\{q_{m}\right\}_{m=1}^{\infty}$ (without loss of generality we may assume that this is $\left\{q_{m}\right\}_{m=1}^{\infty}$ itself) which converges to a function $F$ uniformly on $[0,1-\varepsilon / 2]$. Then, by the Clarkson-Erdös Theorem of the Introduction, $F$ is analytic on $(0,1-\varepsilon / 2)$. On the other hand

$$
|F(x)| \leqq A_{m}^{-1}, \quad x \in[1-\varepsilon, 1-\varepsilon / 2]
$$


and since $A_{m} \rightarrow \infty$, we have

$$
F(x)=0, \quad x \in[1-\varepsilon, 1-\varepsilon / 2]
$$

which implies that $F \equiv 0$ on $[0,1-\varepsilon / 2]$, a contradiction, since $\max _{0 \leqq x \leqq 1-z / 2}|F(x)|=1$. When $0<\lambda_{1}<1$ the scaling $x \rightarrow x^{1 / \lambda_{1}}$ gives the desired result from the already proved case.

Inequality 2 (Remez-type Inequality for Lacunary Systems). If $P \in \operatorname{span}\left\{1, x^{\lambda}, x^{\lambda^{2}}, \ldots\right\}$, $\lambda>1$, and $A \subset[0,1]$ is a closed set of measure at least $\varepsilon>0$, then

$$
|P(0)|<c_{\varepsilon, \lambda}\|P(x)\|_{A}
$$

where $c_{\varepsilon, \lambda}$ depends only on $\varepsilon$ and $\lambda$.

Proof. The extremal polynomial from $M_{n}=\operatorname{span}\left\{1, x^{\lambda}, x^{\lambda^{2}}, \ldots, x^{\lambda^{n}}\right\}$ is, by a simple perturbation argument, just the Chebyshev polynomial $T_{n}$ on $A$. By Lemmas 8 and 9 this polynomial is bounded on an interval of length $c_{\lambda, \varepsilon}>0$ (independently of $A$ and $n$ ) in $[0,1]$. The rest now follows from Inequality 1 in the Interval Case.

\section{Comparison theorems}

The following comparison theorem holds and shows that the Remez constant gets smaller as the Müntz system gets sparser.

Theorem 2. Suppose $\left\{\lambda_{i}\right\}$ and $\left\{\gamma_{i}\right\}$ are increasing sequences of positive real numbers. If

$$
\lambda_{i} \leqq \gamma_{i}, \quad \lambda_{0}=\gamma_{0}=0
$$

and $A$ is a compact set in $(\rho, \infty)$, where $\rho>0$. Then

$$
\sup _{\left\{a_{i}\right\}} \frac{\left\|\sum_{i=0}^{n} a_{i} x^{\lambda_{i}}\right\|_{[0, \rho]}}{\left\|\sum_{i=0}^{n} a_{i} x^{\lambda_{i}}\right\|_{A}} \geqq \sup _{\left\{b_{i}\right)} \frac{\left\|\sum_{i=0}^{n} b_{i} x^{\gamma_{i}}\right\|_{[0, \rho]}}{\left\|\sum_{i=0}^{n} b_{i} x^{\gamma_{i}}\right\|_{A}}
$$

Proof. A simple perturbation argument shows that the extremal polynomial for

$$
\max _{\left(b_{i}\right)} \frac{\left\|\sum_{i=0}^{n} b_{i} x^{\gamma_{i}}\right\|_{[0, \delta]}}{\left\|\sum_{i=0}^{n} b_{i} x^{\gamma_{i}}\right\|_{A}}
$$

is just the Chebyshev polynomial $T_{n}$ on $A$ from $\operatorname{span}\left\{1, x^{y_{1}}, \ldots, x^{\gamma_{n}}\right\}$. If not, it would be possible to increase the value of $p(y):=\sum_{i=0}^{n} a_{i} y^{\lambda_{i}}$ for every fixed $0 \leqq y \leqq \rho$ without increasing $\|p(x)\|_{A}$. Now observe that for $T_{n}$ 


$$
\left\|T_{n}\right\|_{[0, \rho]}=\left|T_{n}(0)\right|
$$

because $T_{n}$ and hence $T_{n}^{\prime}$ has no zeros on $(0, \rho)$.

Now let $R_{n} \in \operatorname{span}\left\{1, x^{\lambda_{1}}, \ldots, x^{\lambda_{n}}\right\}$ interpolate $T_{n}$ at the zeros of $T_{n}$, and be normalized so that $R_{n}(0)=T_{n}(0)$. Theorem 1 of [15], now gives

$$
\left|R_{n}(x)\right| \leqq\left|T_{n}(x)\right|, \quad x \in A
$$

and the result follows.

Proofs of Theorem 1 and Inequality 1 . Inequality 1 is immediate from the above theorem and Inequality 2, while Theorem 1 with the remark right after it now follows from Inequality 1 and the results of Clarkson and Erdös given in the Introduction.

\section{An example}

Theorem 3. Let $\left\{\lambda_{i}\right\}$ be an arbitrary sequence of distinct positive real numbers. Then there exists a non-empty perfect set $E \subset[0,1]$ and Müntz polynomials $P_{m} \in$ span $\left\{x^{\lambda_{0}}, x^{\lambda_{1}}, \ldots\right\}$ such that $\left\|P_{m}\right\|_{E} \leqq 1$ and $\left|P_{m}(0)\right| \rightarrow \infty$ when $m \rightarrow \infty$.

Proof. Let $M_{n}:=\operatorname{span}\left\{x^{\lambda_{0}}, x^{\lambda_{1}}, \ldots, x^{\lambda_{n}}\right\}$. Choose two distinct points $x_{1,1}$ and $x_{1,2}$ in $(0,1)$, By interpolating we can find a $P_{1} \in M_{2}$ such that

$$
P_{1}\left(x_{1,1}\right)=P_{1}\left(x_{1,2}\right)=0 \text { and } P_{1}(0)=1 .
$$

Choose two disjoint closed intervals $E_{1,1}$ and $E_{1,2}$ so that $x_{1,1} \in E_{1,1}, x_{1,2} \in E_{1,2}$ and

$$
\left|P_{1}(x)\right| \leqq 1 \quad \text { for every } \quad x \in E_{1,1} \cup E_{1,2} .
$$

Assume that a sequence of Müntz polynomials $\left\{P_{j}\right\}_{j=1}^{m}$ and closed intervals $E_{j, k}$ $\left(1 \leqq j \leqq m, 1 \leqq k \leqq 2^{j}\right)$ have already been constructed so that the intervals $E_{j, k}\left(1 \leqq k \leqq 2^{j}\right)$ are pairwise disjoint for every fixed $j(1 \leqq j \leqq m), E_{j+1,2 i-1} \subset E_{j, i}$ and $E_{j+1,2 i} \subset E_{j, i}$ $\left(1 \leqq j \leqq m-1,1 \leqq i \leqq 2^{j-1}\right),\left|P_{j}(x)\right| \leqq 1$ on each $E_{j, k}\left(1 \leqq j \leqq m, 1 \leqq k \leqq 2^{j}\right)$ and $P_{j}(0)=2^{j}$ $(1 \leqq j \leqq m)$. Take two distinct points $x_{m+1,2 i-1}$ and $x_{m+1,2 i}$ from each $E_{m, i}\left(1 \leqq i \leqq 2^{m}\right)$. By interpolating we can find a $P_{m+1} \in M_{2^{m+1}}$ such that

$$
P_{m+1}\left(x_{m+1, k}\right)=0, \quad 1 \leqq k \leqq 2^{m+1} \text { and } P_{m+1}(0)=2^{m+1} .
$$

For every $i\left(1 \leqq i \leqq 2^{m}\right)$ choose two disjoint closed intervals $E_{m+1,2 i-1}$ and $E_{m+1,2 i}$ such that $x_{m+1,2 i-1} \in E_{m+1,2 i-1}, x_{m+1,2 i} \in E_{m+1,2 i}, E_{m+1,2 i-1} \cup E_{m+1,2 i} \subset E_{m, i}$ and

$$
\left|P_{m+1}(x)\right| \leqq 1 \quad \text { for every } \quad x \in \bigcup_{k=1}^{2^{m+1}} E_{m+1, k}
$$


Now let $E:=\bigcap_{m=1}^{\infty} \bigcup_{k=1}^{2^{m}} E_{m, k}$. Obviously $E$ is perfect, $P_{m} \in M_{2^{m}}$ and $P_{m}(0)=2^{m}$. Thus the theorem is proved.

\section{Countable sets}

Some of the subtleties of Müntz's theorem on subsets are illustrated by the following pair of theorems.

Theorem 4. Let $\left\{\lambda_{i}\right\}$ be an arbitrary sequence of distinct positive real numbers. Then there exists a closed infinite set (a convergent sequence with its limit) $S \subset[0,1]$ such that span $\left\{1, x^{\lambda_{1}}, x^{\lambda_{2}}, \ldots\right\}$ is dense in $C[S]$ in the uniform norm.

Proof. Let $y_{1}=1 / 2$ and assume that $\left\{y_{j}\right\}_{j=1}^{n} \subset(0,1)$ has already been constructed. We choose a $y_{n+1}$ such that

(1) $y_{n}<y_{n+1}<1$

(2) $1-(n+1)^{-1}<y_{n+1}$,

(3) $|p(x)| \leqq(n+1)^{-1}$ for every $p \in M_{n}:=\operatorname{span}\left\{1, x^{\lambda_{1}}, \ldots, x^{\lambda_{n}}\right\}$ satisfying $\left|p\left(y_{j}\right)\right| \leqq 1$, $j=1,2, \ldots, n$, and $p(1)=0$, and for every $y_{n+1} \leqq x \leqq 1$.

The existence of such a $y_{n+1}$ follows from the following argument. In $M_{n}$ we define the norms

$$
\|p\|_{1}:=\sum_{j=0}^{n}\left|a_{j}\right| \text { and }\|p\|_{2}:=|p(1)|+\sum_{j=1}^{n}\left|p\left(y_{j}\right)\right|
$$

of a Müntz polynomial $p(x)=a_{0}+\sum_{j=1}^{n} a_{j} x^{\lambda_{j}}$ which are equivalent to each other. If $p \in M_{n},\left|p\left(y_{j}\right)\right| \leqq 1(j=1,2, \ldots, n)$ and $p(1)=0$, then $\|p\|_{2} \leqq n$, hence $\|p\|_{1} \leqq K$ with some constant $K$ depending only on $n$. Therefore there is a constant $K^{\prime}=K^{\prime}(n) \geqq 1$ such that $\max _{1 / 2 \leqq x \leqq 1}\left|p^{\prime}(x)\right| \leqq K^{\prime}$ for every $p \in H_{n}$, which, together with the Mean Value Theorem and $p(1)=0$, implies

$$
|p(x)|=|p(1)-p(x)|=(1-x)\left|p^{\prime}(\xi)\right| \leqq(1-x) K^{\prime} \leqq(n+1)^{-1}, \quad p \in M_{n}
$$

if $1-x \leqq\left((n+1) K^{\prime}\right)^{-1}$. Hence

$$
y_{n+1}:=\max \left\{y_{n}+\frac{1-y_{n}}{2}, 1-\left((n+1) K^{\prime}\right)^{-1}\right\}
$$

is suitable. Obviously $\lim _{n \rightarrow \infty} y_{n}=1$. Let $S:=\left\{y_{n}\right\}_{n=1}^{\infty} \cup\{1\}$. We show that $\operatorname{span}\{1$, $\left.x^{\lambda_{1}}, x^{\lambda_{2}}, \ldots\right\}$ is dense in $C[S]$. Let $f$ be continuous on $S$, without loss of generality we may assume that $f(1)=0$. Let $L:=\max _{x \in S}|f(x)|$. Choose $p_{n} \in M_{n}(n=1,2, \ldots)$ such that 
$p_{n}\left(y_{j}\right)=f\left(y_{j}\right)(j=1,2, \ldots, n)$ and $p_{n}(1)=0$ (this is the interpolation property of a Haar space). Then, from the choice of $\left\{y_{j}\right\}_{j=1}^{\infty}$ we easily deduce that

$$
\max _{x \in S}\left|f(x)-p_{n}(x)\right| \leqq \frac{L}{n+1}
$$

which proves the theorem.

Our next theorem, together with Müntz's and Tietze's theorems, will show that if $\left\{\lambda_{i}\right\}$ is a sequence of real numbers satisfying $\inf _{i}\left(\lambda_{i+1}-\lambda_{i}\right)>0$, then $\operatorname{span}\{1$, $\left.x^{\lambda_{1}}, x^{\lambda_{2}}, \ldots\right\}$ is dense in $C[S]$ in the uniform norm for every countable closed $S \subset[0,1]$ if and only if $\sum_{i=1}^{\infty} 1 / \lambda_{i}=\infty$.

Theorem 5. Let $\left\{\lambda_{i}\right\}_{i=1}^{\infty}$ be a sequence of positive real numbers such that $\sum_{i=1}^{\infty} 1 / \lambda_{i}<\infty$ and $\inf _{i}\left(\lambda_{i+1}-\lambda_{i}\right)>0$. Then there is a countable closed subset $S$ of $[0,1]$ so that $\operatorname{span}\left\{1, x^{\lambda_{1}}, x^{\lambda_{2}}, \ldots\right\}$ fails to be dense in $C[S]$ in the uniform norm.

Proof. For every $n \in \mathbb{N}$, let $E_{n}$ be the collection of the $n+1$ extreme points of the $n$th Chebyshev polynomial $T_{n}=T_{n}\left\{\left[0, \lambda_{1}, \ldots, \lambda_{n}\right]:[0,1]\right\}$ of $M_{n}$ and let $E=\cup_{n=1}^{\infty} E_{n}$. Since $\sum_{i=1}^{\infty} 1 / \lambda_{i}<\infty$, Lemma 4 gives $0<c:=\inf \{E \backslash\{0\}\}$, hence we can choose three points $0<y_{1}<y_{2}<y_{3}<c$. Now let $S=\bar{E} \cup\left\{y_{1}, y_{2}, y_{3}\right\}$. By an observation of [1] we have $E^{\prime \prime}=\{1\}$, where $E^{\prime}$ denotes the collection of the limit points of $E$. Therefore $S^{\prime \prime}=\{1\}$ as well, hence $S$ is a closed countable set. Now let $f$ be continuous on $S, f(x)=0$ on $[c, 1]$, $f\left(y_{1}\right)=f\left(y_{3}\right)=2$ and $f\left(y_{2}\right)=-2$. Assume that there is a $p \in M_{n}$ such that $\max _{x \in S} \mid p(x)-$ $f(x) \mid \leqq 1 / 2$. Then it is easy to check that $p-T_{n} \in M_{n}$ has at least $n+1$ zeros in $(0,1)$, which is a contradiction. This finishes the proof.

We remark that if $\left\{\lambda_{i}\right\}$ is an arbitrary sequence of distinct positive real numbers then there is a non-empty perfect set $S \subset[0,1]$ such that $\operatorname{span}\left\{x^{\lambda_{1}}, x^{\lambda_{2}}, \ldots\right\}$ is dense in $C[S]$ in the uniform norm. This can be obtained by straightforward modifications of the proof of Theorem 3.

\section{REFERENCES}

1. P. B. Borwein, Zeros of Chebyshev polynomials in Markov systems, J. Approx. Theory 63 (1990), 56-64.

2. P. B. Borwein and E. SAFF, On the denseness of weighted incomplete approximations, in A. A. Gonchar and E. B. Saff (Eds.), Progress in Approximation Theory (Springer-Verlag, 1992), 419-429.

3. P. B. Borwein, Variations on Müntz's theme, Canad. Math. Bull. 34 (1991), 305-310.

4. E. W. CheneY, Introduction to Approximation Theory (McGraw-Hill, New York, 1966).

5. J. A. Clarkson and P. Erdös, Approximation by polynomials, Duke Math J. 10 (1943), 5-11. 
6. T. ERdELYI, Remez-type inequalities on the size of generalized polynomials, J. London Math. Soc. 45 (1992), 255-264.

7. R. P. Feinerman and D. J. Newman, Polynomial Approximation (Williams and Wilkins, Baltimore, MD, 1976).

8. M. von Golitschek, A short proof of Müntz's theorem, J. Approx. Theory 39 (1983), 394-395.

9. I. I. Hirschman, JR., Approximation by non-dense sets of functions, Ann. of Math. 50 (1949), 666-675.

10. D. Leviatan, Improved estimates in Müntz-Jackson theorems, manuscript.

11. P. C. McCarthy, J. E. Sayre and B. L. R. Shawyer, Generalized Legendre polynomials, manuscript.

12. D. J. Newman, Derivative bounds for Müntz polynomials, J. Approx. Theory 18 (1976), $360-362$.

13. D. J. Newman, Approximation with rational functions, Regional Conference Series in Mathematics 41 (1978).

14. L. Schwartz, Etude des Sommes d'Exponentielles (Hermann, Paris, 1959).

15. P. W. Sмrrr, An improvement theorem for Descartes systems, Proc. Amer. Math. Soc. 70 (1978), 26-30.

16. T. T. Trent, A Müntz-Szász theorem for $C(D)$, Proc. Amer. Math. Soc. 83 (1981), 296-298.

Department of Mathematics

DALHOUSIE UNIVERSITY

Halifax, Nova Scotia

CANADA, B3H $3 \mathrm{~J} 5$
Department of Mathematics

The Ohio State University

Columbus, Оhıо 43210

USA 